\title{
光外差-Zeeman 调制磁旋转光谱技术
}

\section{陈扬骎 毕志毅 蔡佩佩 丁晶新 王荣军 卢晶晶 马龙生}

(华东师范大学物理系国家教育部量子光学开放实验室, 上海 200062)

摘要 介绍采用光外差技术与 Zeeman 调制磁旋转技术相结合, 形成一种高信噪比、高灵敏度 和无吸收本底的光谱技术, 可以消除来自光源的幅度涨落噪声, 达到散粒噪声的测量极限。

\section{关键词 光外差光谱 磁旋转光谱 瞬态分子}

顺磁性分子是具有不配对电子的一类分子, 如许多自由基分子、激发态分子和开壳层离子 分子, 它们的 电子自旋角动量或电子轨道角动量不为零, 因而在外场中的 Zeeman 效应较强, 其量子结构和光谱结构也相当复杂, 因此对这类分子的研究始终是分子物理学基础研究和应 用研究的前沿课题. 通常顺磁性分子的化学活性很强、寿命很短, 也常被称为瞬态分子. 这些 分子在实验室条件下的生成浓度极低, 它们的光谱测量就比较困难, 尤其是对它们的吸收光谱 测量更为困难. 然而在许多应用场合, 吸收光谱方法又是非常重要的测量手段, 因此需要发展 高灵敏度吸收光谱技术来适应这方面的研究.

本文提出一种新的高灵敏度吸收光谱技术: 光外差-Zeeman 调制磁旋转光谱技术( Optical heterodyne zeeman modulation magnetic rotation spectroscopy, 简称为 $\mathrm{OH}-\mathrm{ZM}-\mathrm{MRS})$, 这是在 Field 等人 ${ }^{[1]}$ 所采用的频率调制-磁旋转光谱方法(简称为 FM-M RS) 的基础上, 采用调制频率 更高的光外差光谱技术与交流磁场调制的磁旋转光谱技术相结合, 形成一种高信噪比、高灵敏 度和无吸收本底的光谱技术, 并以 $\mathrm{N}_{2}$ 分子激发态跃迁谱线为测量对象给出实验的结果.

\section{1 基本原理和实验装置}

光外差光谱技术和 Zeeman 调制-磁旋转光谱技术 (Zeeman modulation-magnetic rotation spectroscopy 以下简称 ZM-M RS) 都分别在文献 $[2,3]$ 中做了报道, 在此我们对两种光谱技术 分别作简单的叙述.

Zeeman 调制-磁旋转光谱方法是利用顺磁性分子样品在纵向磁场中的 Faraday 效应, 对入 射线偏振光产生偏振面的旋转或椭偏效应, 通过吸收池后的透射光, 由检偏器检测磁旋光信 号, 该信号可以由下式近似表示 ${ }^{[4]}$ :

$$
I=I_{0}\left|\varphi^{2}-\varphi^{2} k_{0} l\left(k_{+}+k_{-}\right)+\varphi_{k_{0}} l\left(n_{+}-n_{-}\right)\right|,
$$

式中 $I_{0}$ 和 $k_{0}$ 分别为入射光的强度和波矢, $l$ 为吸收池程长, $k_{+}, k_{-}$和 $n_{+}, n_{-}$为样品对入射 线偏振光所分解的左旋光和右旋光的吸收率和折射率. $\varphi$ 为吸收池前后一对偏正器偏离正交 的一个非常小的弧度角. 这种方法的信噪比由下式决定 ${ }^{[5]}$ :

$$
\frac{S}{N}=\frac{R_{\Delta} l \varphi}{\varphi^{2}+\zeta}
$$

其中 $R_{\Delta}$ 是正比于 $\left(n_{+}-n_{-}\right)$的函数. 达到最佳信噪比时所要求的 $\varphi= \pm \sqrt{\zeta}, \zeta$ 为偏振器的 消光比. 由式(2) 可知偏振器的消光比越好, 则信噪比越高. 由于 $\varphi \ll 1$, 因此式(1) 中与 $\varphi^{2}$ 成 
正比的第 1 项(代表光源本底信号)和第 2 项(代表吸收项)都可以忽略, 信号主要来源于磁旋 光的第 3 项. 利用这种方法可以使探测器在暗背景条件下工作, 减少了吸收本底. 此外, 所加 的交流调制磁场实质上是通过分子的 Zeeman 效应对 $n_{+}$和 $n_{-}$进行调制, 是一种对分子谱线 自身进行调制的光谱方法, 因而在很大程度上可以避免外来噪声和杂散调制的干扰. 但这种 方法受到调制磁场驱动功率源的限制, 调制频率不可能做得很高, 通常小于 $1 \mathrm{kHz}$; 又由于偏 振器消光比的限制, 在这个调制频率范围内, 入射激光光源的幅度涨落噪声仍是探测系统噪声 的主要来源, 从而限制了测量灵敏度.

光外差光谱技术是采用电光晶体在激光腔外对光源作位相调制, 在光源主频两边产生对 称分布的边频. 在调制度较小的情况下, 经调制后的光源电矢量可用下式表达:

$$
\boldsymbol{E}=\boldsymbol{E}_{0}\left\{J_{0}(\beta) \sin \Omega_{t}+J_{1}(\beta)\left[\sin (\Omega+\omega) t-\sin \left(\Omega_{-} \omega\right) t\right]\right\},
$$

式中 $\Omega$ 为激光频率, $\omega$ 为电光晶体的射频调制频率, $J_{0}(\beta)$ 和 $J_{1}(\beta)$ 均为 Bessal 函数, $\beta$ 为调制 度. 当该光束直接入射到探测器时, 正负边频与主频均可在探测器上产生频率为 $\omega$ 的拍频电 流. 但由于正负边频的幅度相等, 位相相反, 探测器无拍频电流输出. 如果该光束通过样品池 后, 由于样品的吸收和色散, 上述平衡被破坏, 探测器在 $\omega$ 处会有拍频电流输出, 它包含有样 品对入射光的吸收和色散信息. 当调制频率超过 $2 \mathrm{MHz}$ 时, 在探测系统上激光光源的幅度涨 落噪声可以降至散粒噪声的极限. 在我们的光外差光谱测量中, 电光晶体调制频率采用远大 于 $2 \mathrm{MHz}$ 的射频频率, 因而可以极大地抑制来自光源的噪声, 弥补了 Zeeman 调制-磁旋转光 谱调制频率过低的缺陷. 但在使用电光晶体作位相调制的过程中, 不可避免地有剩余的幅度 调制效应, 因而在一定程度上测量仍然会受到光源幅度涨落噪声的影响; 此外, 吸收池的窗片 和某些光学器件经常形成偶然的 F-P 干涉效应. 在光源频率调谐过程中. 将会产生周期性幅度 波动噪声, 这个问题恰好可以由 Zeeman 调制-磁旋转光谱方法来克服.

我们将这两种方法结合起来, 形成一种双调制的高灵敏度光谱方法: 光外差-Zeeman 调 制磁旋转光谱( OH-ZM-M RS) 方法, 实验方框图如图 1 所示.

用 $\mathrm{Ar}$ 离子激光洜浦染料激光器输出的稳频单模激光束作为光源, 其线宽为 $1 \mathrm{MHz}$, 功率 为 $50 \mathrm{~mW}$, 经 P1 偏振器 (本实验中的偏振器均采用 Glan-T aylor 棱镜, 消光比优于 $10^{-5}$ ) 起偏 后, 入射到铌酸锂晶体 $(3 \mathrm{~mm} \times 3 \mathrm{~mm} \times 20 \mathrm{~mm})$ 构成的电光调制器中进行位相调制. 由于吸收 样品 $\mathrm{N}_{2}$ 分子在可见光波段中的 Dopplor 线宽约为 $1 \mathrm{GHz}$, 在本实验中采用了等于谱线半宽度 的调制频率, 约为 $500 \mathrm{MHz}$, 以获得最佳信号. 为了保证入射到吸收池的激光束有良好的线偏 振特性, 吸收池前再加上 P2 偏振器. 吸收池由 $\mathrm{i} 15 \times 400 \mathrm{~mm}$ 圆柱型玻璃管和石英窗片构成. 吸收池内两端各有一个圆筒型镍电极, 用它对流动纯氮气进行放电, 以产生激发态氮分子, 放 电电流为 $15 \mathrm{~mA}$. 整个吸收池置于一个螺线管磁场线圈中, 该线圈由 $375 \mathrm{~Hz}$ 音频功率源驱 动, 可产生峰值为 $40 \mathrm{mT}$ 的交流调制磁场, 用于对 $\mathrm{N}_{2}$ 分子跃迁谱线的 Zeeman 调制. 通过吸 收池的激光束由与 P2 近正交的 P3 偏振器进行检偏, 经 $560 \mathrm{~nm}$ 滤光片滤除放电产生的杂散 光后由雪崩光电二极管进行探测, 其输出经 $500 \mathrm{MHz}$ 选频前置放大器放大后由平衡混频器检 出 $500 \mathrm{MHz}$ 拍频信号, 再由锁相放大器检出 $375 \mathrm{~Hz}$ 磁旋转信号. 最后由计算机系统进行数 据采集和处理. 激光频率由波长计和 $\mathrm{NO}_{2}$ 参考吸收谱线进行标定, 准确度优于 $0.005 \mathrm{~cm}^{-1}$, 波长标定部分未画入图 1 中, 可参考文献[6]. 


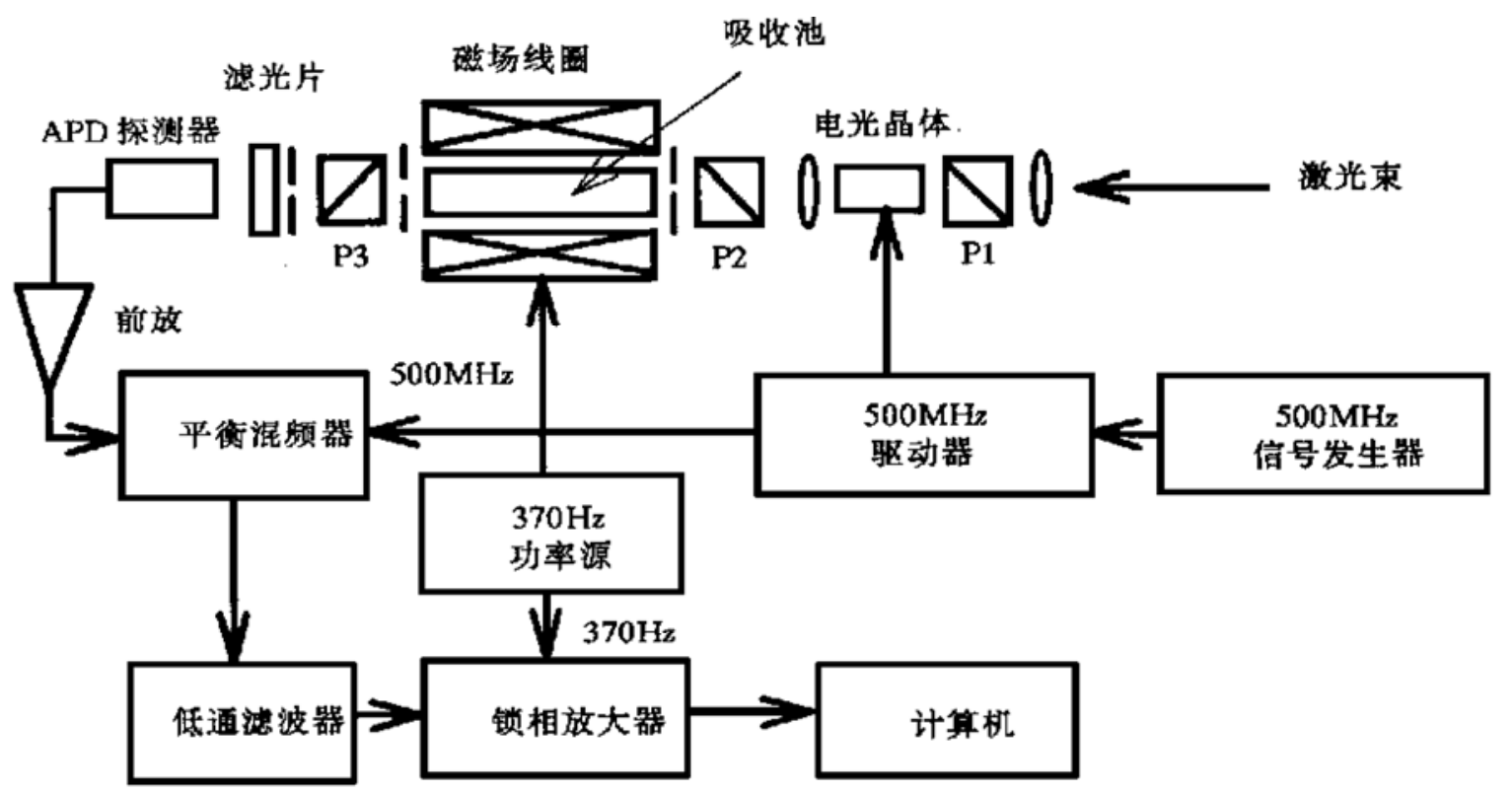

图 1 光外差-Zeeman 调制磁旋转光谱实验方框图

\section{2 结果与讨论}

采用光外差 -Zeeman 调制磁旋转光谱方法对 $\mathrm{N}_{2}$ 分子第 1 正带 $B^{2} \prod_{\mathrm{g}} \leftarrow \mathrm{A}^{3} \Sigma_{\mathrm{u}}^{+}$在 $17132 \mathrm{~cm}^{-1}$ 附近 的跃迁谱线进行测量 ${ }^{[7]}$, 激光一次扫描范围为 $1 \mathrm{~cm}^{-1}$. 图 2 为分别采用 Zeeman 调制磁旋转光谱 方法和光外差-Zeeman 调制磁旋转光谱方法对 $\mathrm{N}_{2}$ 谱线的测量结果.

从图 2 中可以看到以下几点:

(1) 光外差-Zeeman 调制磁旋转光谱方法, 由 于采用了 $500 \mathrm{MHz}$ 光位相调制和 $375 \mathrm{~Hz}$ 磁调制的 双频调制技术, 并通过光外差技术探测分子的磁旋 转光谱信号, 有效地抑制了光源的幅度涨落噪声、 背景噪声和由电光调制器剩余幅度调制所引入的 噪声, 因而信噪比与 Zeeman 调制-磁旋转光谱的测 量结果相比有了很大的提高. 由于实验中使用的 数字式模拟信号采集系统的位数还不够高, 因而在 图 2 的 2,3 情况中, 测量信噪比受到数据采集系统 数字噪声的限制, 实际的信噪比比图 2 的 2,3 所显 示的还要高.

(2) Zeeman 调制-磁旋转光谱方法中, 当 $\varphi=0$ 时, 即 P2 和 P3 偏振器完全正交的情况下, 信号几
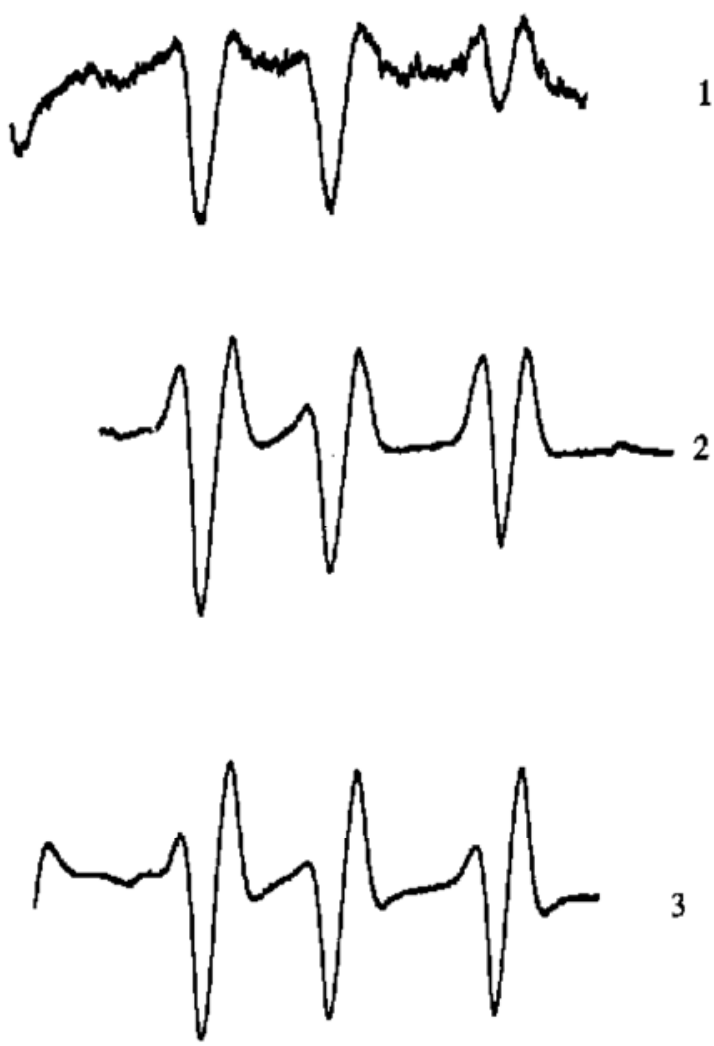

图 $2 \mathrm{~N}_{2}$ 第 1 正带跃迁谱线

1 -Zeeman 调制磁旋转光谱(ZM-M RS) 方法测量结 果, $\varphi \neq 0 ; 2,3$ 一光外差-Zeeman 调制磁旋转光谱 方法测量结果, 2 为 $\varphi=0$ 的情况, 3 为 $\varphi \neq 0$ 的情况 
乎为零, 只有当 $\varphi$ 为最佳角度时 (即 $\varphi= \pm \sqrt{3}$ ), 有较强的二次微分线型信号, 此时光源的幅度 噪声也伴随出现在信号中. 然而, 在光外差-Zeeman 调制磁旋转光谱测量中, 无论 $\varphi=0$ 或 $\varphi \neq 0$ 时, 都能获得信噪比很好的信号, 尤其在 $\varphi=0$ 的情况下, 由于 P2 和 P3 偏振器处于完全 正交状态, 对噪声和本底的抑制更为有利.

(3) 实验还表明光外差-Zeeman 调制磁旋转光谱测量对外界杂散光的抗干扰能力大大地 增强了, 甚至可以在室内照明开启状态下进行工作; 而 Zeeman 调制-磁旋转方法只能在暗室 条件下进行测量.

从以上结果表明光外差-Zeeman 调制磁旋转光谱技术能综合光外差光谱方法和 Zeeman 调制-磁旋转光谱方法的特点, 可以消除来自光源的幅度涨落噪声, 实现无吸收本底的测量, 极 大地提高了测量信噪比. 同时又保留了 Zeeman 调制-磁旋转光谱技术的许多特点 ${ }^{[6]}$, 即可以 对分子的转动谱带结构产生一定程度的简化作用, 以及利用谱线线型和相位上的特征, 非常方 便地对复杂的分子转动谱带结构进行正确的指认.

光外差-Zeeman 调制磁旋转光谱方法的对象局限于顺磁性分子, 因为只有顺磁性分子才 有较强的 Zeeman 效应, 可以通过磁场的调制来获得信号, 因此这种方法可以避免大量非顺磁 性分子光谱线的干扰. 由于这种光谱方法有很高的测量灵敏度, 可以用来对许多瞬态顺磁性 分子的吸收光谱进行测量. 此外, 该方法也可以对一些抗磁性分子的激发态进行测量, 如 $\mathrm{N}_{2}$ 分子的基态是非顺磁性的 ${ }^{1} \sum$ 态, 但其激发态由于出现电子自旋角动量或电子轨道角动量而具 有顺磁特性. 这种适合于瞬态分子的高灵敏度吸收光谱方法将可以应用于化学反应动力学、 燃烧物理学、等离子体物理学、污染监测和环境科学等多方面的研究领域.

致谢 本工作为国家自然科学基金(批准号：19574015) 和波谱和原子分子物理国家重点实验室( 批准号: 961512) 资助项目.

\section{参 考 文 献}

1 McCarthy M C, Field R W. Frequency-modulation enhanced magnetic rotation spectroscopy of PdH. J Chem Phys, 1994, 100(9): 6347

2 夏长平, 陈扬椿, 蔡佩佩, 等. 塞曼调制磁旋转分子光谱特征的研究. 原子与分子物理学报, 1997, 14(4)：547

3 Hall J L, Hollberg L, Ma L S, et al. Progress tow ard phase stable optical frequency standards. J Phys (France) Colloq, 1981, 42(1): 59

4 Hinz A, Pfeiffer J, Bohle W, et al. M id infrared laser magnetic resonance using the Faraday and Voigt effects for sensitive detection. Mol Phys, 1982, 45(6): 1131

5 Litfin G, Pollick C R, Curl R F. Sensitivity enhancement of laser absorption spectroscopy by magnetic rotation effect. J Chem Phys, 1980, 72(12): 6602

6 陈金海, 陈杨椿, 蔡佩佩, 等. 塞曼调制磁旋转激光光谱方法. 光学学报, 1996, 16( 5): 612

7 王 辉, 蔡佩佩, 夏长平, 等. $\mathrm{N}_{2}$ 第一正带的塞曼调制磁旋转光谱分析. 化学物理学报, 1997, 10(2): 117

(1998-07-03 收稿, 1998-10-12 收修改稿)

更正 本刊 1998 年第 23 期第 2545 页韩宝福等人一文第二节中“表 2 是各种矿物的代表性化学成分.”一句话应删掉, 特向作者和读者致兼. 